\title{
Genetic Counseling and Assisted Reproductive Technologies
}

\author{
Debra Lilienthal ${ }^{1}$ and Michelle Cahr ${ }^{2}$ \\ ${ }^{1}$ The Ronald O. Perelman and Claudia Cohen Center for Reproductive Medicine, Weill Cornell Medicine, \\ New York, New York 10021, USA \\ ${ }^{2}$ California Cryobank Life Sciences, Los Angeles, California 90025, USA \\ Correspondence: dp19001@med.cornell.edu
}

\begin{abstract}
Despite the ever-increasing number of patients undergoing fertility treatments and the expanded use of genetic testing in this context, there has been limited focus in the literature on the involvement of genetics professionals in the assisted reproductive technology (ART) setting. Here we discuss the importance of genetic counseling within reproductive medicine. We review how genetic testing of embryos is performed, the process of gamete donation, the challenges associated with genetic testing, and the complexities of genetic test result interpretation.
\end{abstract}

In 2018, Louise Brown, the first child born from in vitro fertilization (IVF), turned $40 \mathrm{yr}$ old (Steptoe and Edwards 1978; Fishel 2018). For people who were previously unable to have a biological child, the ability to conceive via IVF meant that their dream of parenthood had a greater chance of becoming a reality. IVF has come a long way over the last four decades, and the number of children born following the use of assisted reproductive technology (ART) continues to grow rapidly. In 2016 alone, data from the Centers for Disease Control and Prevention reported 263,577 IVF cycles, resulting in 76,930 total infants born; the number of IVF cycles documented between January 9, 1969 and August 1, 1978 was 457, resulting in two live births (Centers for Disease Control and Prevention 2018; Fishel 2018). Given the societal trends of increasing parental age and delayed child- bearing, both of which correlate with reduced fertility, these numbers are expected to continue to rise (Botting and Dunnell 2000; Armstrong and Akande 2013).

Advances in genetic testing have become increasingly intertwined with the world of IVF, and are critical components of reproductive medicine. Given the complexity of these advances, the inclusion of trained genetics professionals within the field of ART is vital.

\section{BACKGROUND}

There are many reasons for individuals and couples to seek out ART, including female infertility, male factor infertility, unexplained infertility, secondary infertility (infertility after a previous successful pregnancy), and the use of a gestational carrier. Some who choose to use

Editors: Laura Hercher, Barbara Biesecker, and Jehannine C. Austin

Additional Perspectives on Genetic Counseling: Clinical Practice and Ethical Considerations available at

www.perspectivesinmedicine.org

Copyright (C) 2020 Cold Spring Harbor Laboratory Press; all rights reserved; doi: 10.1101/cshperspect.a036566

Cite this article as Cold Spring Harb Perspect Med 2020;10:a036566 
ART do not meet the traditional definition of infertility (i.e., not conceiving after 6 or 12 months of unprotected intercourse, depending on age); this includes individuals wishing to prevent genetic disease, same-sex couples, or single patients who require donor gametes, those pursuing sex selection, and those interested in fertility preservation. Fertility preservation may be used when health-related issues or medical treatments affect future fertility, such as gonadotoxic treatments for cancer or delayed childbearing. The reasons for seeking treatment will likely continue to change over time.

Before initiating any form of treatment, American Society for Reproductive Medicine (ASRM) guidelines suggest a thorough evaluation including a physical exam and laboratory testing (Practice Committee of the American Society for Reproductive 2015a,b). This evaluation should elicit personal and family history of genetic disease and review any prior genetic test results (i.e., chromosome analysis, carrier screening, etc.) that could affect the course of treatment. Patients should also be counseled about additional genetic testing that may be indicated before starting treatment. When issues arise as a result of this evaluation, referral to a genetics specialist is recommended.

ART is not synonymous with IVF and lessinvasive treatments may be an appropriate first step in many instances. For some individuals and couples, initial approaches may include timed intercourse or intrauterine insemination (IUI). Reasons to consider IVF as a first-line therapy include the need for any of the following: preimplantation genetic testing (PGT), a gestational carrier, donor oocytes, or intracytoplasmic sperm injection (ICSI) for patients with significant male factor infertility. Regardless of the indication, it is important to note that the IVF process can be physically, emotionally, and financially demanding.

\section{IVF BASICS}

The process of IVF begins with ovarian stimulation. Injectable gonadotropin medications are given to stimulate the ovaries to produce more mature oocytes than could naturally be pro- duced in one cycle. During this stage, near-daily monitoring by blood and ultrasound occurs in order for the physician to assess the response to treatment and determine when the oocytes are mature and ready for retrieval. When the oocytes appear to be potentially mature, the patient is given medication to trigger ovulation and undergoes surgery to remove the oocytes (egg retrieval). Following retrieval, mature oocytes are fertilized with the designated sperm sample in the laboratory and embryo development is monitored. Embryo(s) are usually transferred into the uterus on the third or fifth day of development. Excess embryos may be cryopreserved for later use.

Oocytes are fertilized either by insemination, in which they are mixed with sperm in a Petri dish, or by ICSI, in which a single sperm is injected into the oocyte. The sperm source may be a fresh ejaculate from the patient's partner, frozen sperm from the patient's partner, or sperm obtained via microsurgical epididymal sperm aspiration (MESA) or testicular sperm extraction (TESE). Alternatively, patients may use frozen sperm from an anonymous donor or a directed donor, who may be a friend or a family member.

ICSI was first used to treat male factor infertility for cases in which IVF by insemination did not lead to fertilization, or when a man had few sperm (Lanzendorf et al. 1988). ICSI is now also used in situations in which there is no male factor infertility, such as with PGT, to reduce the potential paternal contamination of the DNA tested from extraneous sperm that are not fertilizing the oocyte. ICSI is also used to fertilize frozen oocytes or to maximize fertilization if only few or poor-quality oocytes are retrieved.

As mentioned above, following fertilization embryos are monitored for cell division. For patients trying to achieve pregnancy within the cycle, embryo(s) are selected for transfer to the uterus. Experienced embryologists carefully assess embryo quantity and quality to select the best embryo(s) and also determine the day of embryo transfer, which varies from center to center.

Vitrification, a method of rapid freezing, is currently the most widely used technique for cryopreservation, the process of freezing reproductive tissue (Practice Committees of American 
Society for Reproductive and Society for Assisted Reproductive 2013). Some patients choose to cryopreserve all of their suitable embryos without transferring any fresh embryos during the cycle. Patients who require up-front cryopreservation include those who do not have medical clearance to achieve pregnancy, those who are using a gestational carrier, and those who are undergoing PGT or fertility preservation. A frozen embryo transfer (FET) is the process by which a frozen embryo is thawed and transferred into a woman's uterus in a subsequent cycle.

The potential risks to offspring conceived by ART have been debated since its early days. Any potential risks of ART treatments must be carefully weighed against their benefits for individuals and couples who may not otherwise be able to conceive.

In any pregnancy, there is a baseline risk of $3 \%-4 \%$ for a child to have a birth defect or developmental abnormality (Bodurtha and Strauss 2012). Although most babies conceived via ART treatments are healthy, some studies suggest that children born following ART have an increased risk of birth defects compared to those born from spontaneous conceptions (Kurinczuk et al. 2004; Hansen et al. 2005). ICSI, in particular, has been reported in some studies to be associated with an increased incidence of hypospadias, sex chromosome abnormalities, and imprinting defects (i.e., Angelman syndrome, Prader-Willi syndrome, and Beckwith-Wiedemann syndrome) (Alukal and Lamb 2008; Practice Committee of American Society for Reproductive and Practice Committee of Society for Assisted Reproductive 2008). It is unclear if these risks are due to the use of ART itself, possible epigenetic factors, or the use of technologies to overcome infertility that may have a genetic basis, whereby abnormal genetic traits may thus be transmitted to offspring (Alukal and Lamb 2008).

Some patients may delay or decline ART treatment because of their concerns about the possible risks. Additionally, those who are worried about the risks of ICSI may forgo its use, even though it may improve their chances of getting pregnant. For some patients, concerns about the risks following ART may persuade them to request special prenatal diagnostic testing for chromosome abnormalities and/or imprinting defects.

\section{GENETIC TESTING OF EMBRYOS}

\section{Preimplantation Genetic Testing Overview}

PGT involves the removal of one or more nuclei from an oocyte (polar body biopsy) or an embryo (blastomere or trophectoderm cells) to test for mutations or aneuploidy prior to embryo transfer (Practice Committee of the Society for Assisted Reproductive and Practice Committee of the American Society for Reproductive 2008). The purpose of PGT is to reduce the risk of having a child with a genetic disorder or a chromosome abnormality.

There are three types of PGT. PGT for aneuploidy (PGT-A; previously called PGS) is the testing of embryos for chromosome abnormalities. PGT for structural rearrangements (PGT-SR) is the testing of embryos for structural chromosome rearrangements (i.e., translocations and inversions). PGT for monogenic disorders (PGT-M; previously called PGD) is the testing of embryos for single gene defects. PGT requires the use of IVF, oocyte or embryo biopsy, and the transfer of embryo(s) into the uterus based on genetic test results.

A biopsy must be performed to obtain DNA for analysis. Historically, embryo biopsies were performed at the cleavage stage, on day 3 , when embryos have approximately six to eight cells. A single blastomere was removed and analyzed, allowing for a fresh embryo transfer on day 5 post-egg retrieval. After the fresh transfer, excess embryos were considered for cryopreservation for possible future use. Current practice has shifted, and most centers now perform biopsies on day- 5 or day- 6 blastocyst embryos which have more than 100 cells. This type of biopsy allows for more cells to be analyzed. For this biopsy, a sample of cells is taken from the trophectoderm layer of the embryo, which will ultimately become the placenta. In most instances, the embryo is frozen after the biopsy because there is not enough time to obtain PGT results to allow for a fresh embryo transfer. Once results 
are obtained, a woman will return for a FET, by which the desired embryos(s) will be transferred into the uterus. Because they are performed at a later developmental stage, and the number of cells removed is smaller on a percentage basis, blastocyst biopsies are considered less disruptive to the developing embryo than those done at the cleavage stage, while providing more DNA for testing (Practice Committees of the American Society for Reproductive et al. 2018).

Although less commonly performed, a polar body biopsy involves removing the first and second polar bodies from an oocyte. This method can be used to detect maternally inherited mutations or meiotic errors during oocyte development. The results obtained from the polar bodies indirectly reflect the genetic status of the oocyte, as the polar body contains the reciprocal result of what is in the oocyte that would be fertilized to create an embryo. With this method of testing, the paternal contribution cannot be assessed.

The ASRM strongly recommends genetic counseling before starting an IVF cycle for patients considering PGT (Practice Committee of the Society for Assisted Reproductive and Practice Committee of the American Society for Reproductive 2008). The initial genetic counseling consultation should include a discussion of the risk for the individual or couple to have an affected child with a single gene defect and/or a chromosome abnormality, as well as the natural history of the disease. All diseases are not created equal, and not all patients at risk of having a child with a genetic disorder or chromosome abnormality elect to pursue PGT or prenatal testing. All testing options should be discussed, including IVF with PGT, as well as prenatal testing via chorionic villus sampling (CVS) or amniocentesis. The risks, benefits, and limitations of PGT technologies, including the risk for falsepositive and false-negative results, should also be reviewed, and recommendations made for follow-up diagnostic testing during pregnancy.

There are some inherent risks associated with PGT, which is an invasive procedure. One risk is possible damage to the embryo from the biopsy, whereby the embryo arrests and is no longer usable. This risk is generally thought to be low. Extended culture may pose an additional risk; the embryos may not survive in the laboratory until day 5 or 6 for testing or transfer. The biopsy itself may also potentially adversely affect an embryo's ability to implant (Scott et al. 2013). Although initial reports on children born following day-3 biopsies did not show an increased risk for physical or mental disabilities, large, long-term studies, specific to the use of day-5/ 6 biopsy (currently the mostly widely used biopsy technique), have not yet been performed (Liebaers et al. 2010; Heijligers et al. 2018).

Although the goal of PGT is to select normal embryos suitable for transfer, patients should be aware of the issues that may arise with its use. For instance, it is possible that embryos may not reach the appropriate developmental stage to be biopsied. Embryos tested on day 3 may not develop until day 5 for transfer. There also may not be any PGT-normal embryos suitable for transfer. When patients are having a FET of PGT-normal embryos, the embryos may not survive the thaw to be transferred. Moreover, patients should be counseled that PGT-normal embryos do not guarantee pregnancy or a healthy live birth, and that it may take multiple cycles to obtain enough PGT-normal embryos to achieve their family. Because of the complex nature of PGT, patients need to be prepared for the potential outcomes of their test results and have access to a genetics specialist to help interpret them.

\section{Preimplantation Genetic Testing- Aneuploidy}

The purpose of PGT-A is to assess the presence or absence of aneuploidy in order to reduce the risk of transferring chromosomally abnormal embryos and to improve the probability of a successful pregnancy. Aneuploidy is the most common cause of miscarriage and implantation failure (Hassold et al. 1996; Kang et al. 2016; Friedenthal et al. 2018). Currently there are no standard indications for PGT-A (Practice Committee of the Society for Assisted Reproductive and Practice Committee of the American Society for Reproductive 2008). Common reasons for considering its use include, but are not limited to, the following: advanced maternal age 
(AMA), recurrent pregnancy loss (RPL), and recurrent IVF/implantation failure. Additional indications may include patients who have had a prior child or pregnancy with aneuploidy or individuals with a chromosome abnormality who are at an increased risk for having chromosomally abnormal offspring. PGT-A is used for sex selection when there is a risk for sex-linked conditions, sex-specific issues without a clear Mendelian pattern of inheritance (i.e., autism), or family balancing/personal choice. Patients who are undergoing fertility preservation or are using a gestational carrier may elect to undergo PGTA to maximize the success of a future transfer.

PGT-A has been available as an adjunct to IVF since the early 1990s (Verlinsky et al. 1995). Since then, testing techniques have improved, and utilization has expanded significantly. Some studies have shown an increased live birth rate following PGT-A and single embryo transfer (SET) (Dahdouh et al. 2015; Simon et al. 2018), whereas others have only found improvements for certain patient populations, such as those who are of advanced maternal age (AMA) (Kang et al. 2016). Despite this, debates regarding its use remain (Rosenwaks et al. 2018).

PGT-A has evolved significantly since its first use. Initially, PGT-A was performed by fluorescent in situ hybridization (FISH), and it screened for a limited number of chromosome abnormalities via polar body or day-3 biopsy (Delhanty et al. 1993; Munné et al. 1993; Verlinsky et al. 1995). FISH continued to have limitations, such as overlapping fluorescent signals which could lead to misinterpretation of the embryo's chromosome status. Additionally, many chromosome abnormalities were missed because of the small number of chromosomes analyzed and higher incidence of mosaicism in day-3 embryos (Treff et al. 2010; Mertzanidou et al.2013). These issues resulted in false-positive and false-negative results. Many studies also did not demonstrate an improved pregnancy rate with its use (Mastenbroek et al. 2007, 2011).

Because of these drawbacks, there was a desire from experts in the field to move toward molecular technologies, which would eliminate some of the technical limitations of FISH and would allow screening of all 24 chromosomes.
These techniques include array comparative genomic hybridization (aCGH), quantitative polymerase chain reaction (qPCR), single-nucleotide polymorphism (SNP) microarray, and next-generation sequencing (NGS). Although all of these assess aneuploidy, they vary in resolution and depth of read. Newer technologies, such as SNP and NGS, can detect segmental gains and losses of chromosome material. SNP can also detect polyploidies, parental origin of aneuploidy, and uniparental disomy (UPD).

Although they all provide similar results, there may be some circumstances in which a particular technology may prove more beneficial. For instance, triploidy and tetraploidy most frequently result in early miscarriage and are generally considered sporadic with a low risk for recurrence. However, there have been several case reports of women with multiple triploid pregnancies (Pergament et al. 2000; Brancati et al. 2003). In some of these cases, this may be due to an inherited predisposition to meiotic errors (Huang et al. 2004; Filges et al. 2015). Thus, although unlikely, patients with histories of polyploid pregnancies may benefit from the use of SNP microarray for PGT-A. Additionally, for patients with multiple failed fertility treatments and/or miscarriages who are considering the use of donor gametes, determining parental origin of aneuploidy in embryos via SNP microarray may aid in their decision.

Because SNP analysis requires DNA samples from both biological parents, there may be cases in which it is not recommended and/or another PGT-A technology may be more appropriate. Obtaining DNA samples from both biological parents may be challenging when donor gametes are used. However, in some cases, cryobanks may have DNA available from the gamete donor for such purposes. Additionally, consanguinity may complicate SNP analysis because of the high level of shared genetic markers increasing the risk for uninformative results.

The presence of mosaicism may further complicate the interpretation of PGT-A results. Embryonic mosaicism is the presence of two or more genetically different cell types within the same embryo. Mosaicism is very common in human embryos, particularly at the cleavage 
stage (Mertzanidou et al. 2013; Harton et al. 2017). Although less common, mosaicism is still often present in the blastocyst (Fragouli et al. 2011; Harton et al. 2017). Despite trophectoderm biopsies containing several cells, these cells may not be representative of other cells within the trophectoderm and/or inner cell mass. This is the likely explanation for many false-positive and false-negative PGT-A results.

As PGT-A technology has improved, the detection of mosaicism within biopsy samples has become far more common. Previously, results were classified as normal or abnormal. However, a third category has now emerged with the reporting of mosaic results. Although any PGT-A-normal or -abnormal result may theoretically not represent the eventual chromosome makeup of a developing embryo, an initial mosaic result presents a higher level of uncertainty as to an embryo's ultimate fitness.

The decision of whether or not to transfer mosaic embryos can be challenging. Several studies have demonstrated healthy live births following the transfer of mosaic embryos (Greco et al. 2015; Fragouli et al. 2017; Munné et al. 2017; Spinella et al. 2018). Some centers are comfortable transferring these embryos as long as patients have had genetic counseling to discuss the results. Prenatal diagnostic testing is strongly recommended in these cases, and patients should be counseled on the possible outcomes of transfer, including implantation failure, miscarriage, abnormal or mosaic pregnancy detected via prenatal screening/diagnosis, live birth of a child with abnormal or mosaic chromosomes, or a healthy live birth (Harton et al. 2017; Sachdev et al. 2017). A review of mosaic results should distinguish between embryos with abnormalities that have been reported at the time of prenatal diagnosis and live birth versus those that are less likely to be compatible with life. Also, embryos with mosaic chromosome abnormalities associated with an increased risk for UPD need be given special consideration. The uncertainties associated with mosaic results may be viewed by some as a reason to not transfer these embryos.

Because of the limitations of PGT-A technology and the issue of mosaicism, it is important for patients to be counseled that PGT-A is a screening test. Because all screening tests have the potential to give false-positive and falsenegative results, prenatal diagnostic testing should be performed to confirm the chromosome status of a pregnancy following PGT-A (Brezina et al. 2016; Kimelman et al. 2018). In addition, prenatal testing can look for smaller deletions and duplications than PGT-A can detect, while also studying more cells, thus further reducing the risk of undetected mosaicism, although this risk cannot be completely eliminated. Although patients who have undergone IVF may be understandably wary of having invasive diagnostic testing that carries a small risk of miscarriage, they need to be counseled about the differences between screening and diagnostic testing so that they can make a well-informed decision (Devers et al. 2013).

\section{Preimplantation Genetic Testing-Structural Rearrangement}

It is estimated that $\sim 1$ in 560 to 1 in 1100 individuals in the general population carries an apparently balanced structural chromosome rearrangement (Forabosco et al. 2009; Tobler et al. 2014; Simioni et al. 2017). Given that such rearrangements increase the risk for infertility and RPL, it is not uncommon for there to be a higher frequency of these rearrangements found among patients referred for fertility treatment. Some patients may also be identified with structural rearrangements following prenatal diagnosis, analysis of products of conception (POC), birth of a child with an abnormality, or detection in another family member. In others, their rearrangement may have been known since birth, if their mothers underwent CVS or amniocentesis.

For patients who are at an increased risk for unbalanced chromosome products because of a structural rearrangement, PGT-SR allows them to select embryos that have an apparently balanced chromosome complement. Currently, most PGT-SR testing uses the same technology as PGT-A (i.e., NGS or SNP). To assess whether or not PGT-SR is possible, the testing laboratory first needs to review karyotypes. Effectiveness of PGT-SR will depend on the ability of the chosen PGT technology to detect the unbalanced seg- 
ments of the rearrangement. In some cases, such as small deletions or inversions not detectable by NGS or SNP testing, linkage analysis may be necessary, requiring the participation of family members for the development of customized probes.

Although in most cases NGS and SNP are equally effective to screen for unbalanced translocation products, SNP is the preferred technology for carriers of Robertsonian translocations involving chromosomes 14 or 15 because it can detect UPD, which is a concern in these cases.

Because none of the technologies currently available can distinguish between a normal karyotype and a balanced rearrangement, PGT-SR cannot eliminate the risk that a balanced rearrangement is transmitted to the fetus. Research is ongoing to perfect technologies capable of distinguishing between normal and balanced karyotypes (Treff et al. 2011).

\section{Preimplantation Genetic Testing- Monogenic}

PGT-M tests embryos for a specific genetic disorder. There are many reasons why patients may consider this type of testing. Individuals who are affected by and/or have a family history of an autosomal dominant disorder, as well as those who are at risk of having offspring with an autosomal recessive or X-linked condition, are candidates for PGT-M. Others may choose PGT-M for HLA-matching for a child in need of a stem cell transplant, whereas those with antigen sensitivity from a prior pregnancy may use PGT-M to select embryos that are not at risk for hemolytic disease of the newborn.

PGT-M is unique to each patient and requires a custom setup that can take several weeks or months to create. Unlike other genetic tests performed pre- or postnatally from samples in which there are hundreds of cells, PGT-M involves the use of a very small amount of DNA. The DNA is amplified with PCR, and then targeted analysis is performed. Because of the small amount of DNA obtained in an embryo biopsy, PGT-M is prone to allele dropout and amplification failure. To increase the likelihood of getting clear results, linkage analysis is used, which
Genetic Counseling and Assisted Reproduction

can be done with short tandem repeats (STRs) or karyomapping/SNP analysis.

Linkage analysis is based on the concept that genetic markers closer together on a chromosome are more likely to be inherited together. This type of analysis involves participation of family members, usually from a generation above or below the individual or couple undergoing treatment. This can be challenging for those who are trying to keep their fertility treatments private, who have family members who may not be supportive of their decision to do PGT, whose parents are deceased, who are estranged from their families, or who are adopted. For conditions that are autosomal dominant or recessive, DNA from an affected parent or child can be used. For autosomal recessive conditions that are detected by general population carrier screening, testing of the couple's parents is needed to determine which parent carries the trait in order to establish linkage.

For de novo cases in which there is no family history of the disorder, PGT-M may or may not be a viable option. For males with an autosomal dominant de novo disorder, a semen sample can be used to establish linkage because sperm contain a single haplotype. Unfortunately, there is no equivalent way to obtain single cells from a female to establish linkage. Some laboratories will be able to work backward to complete the interpretation and establish linkage by testing the patient's embryos. This method may not permit diagnosis of an embryo as PGT-normal or PGT-abnormal if the embryo yield is low or if there is not a mixture of PGT-affected and PGTunaffected embryos.

Although PGT-M may be performed for multiple genetic disorders, patients must be counseled regarding the likelihood of finding embryos free of these diseases.

\section{SPECIAL TYPES OF PREIMPLANTATION GENETIC TESTING}

\section{Nondisclosing PGT-M}

Nondisclosing PGT-M is used when patients at risk for a serious genetic disease do not want to know their mutation status but wish to ensure 
that no mutation-containing embryos are transferred (Stern et al. 2002). Although it is commonly used for patients at risk for Huntington's disease, nondisclosing PGT-M can be used for any disease for which a prospective parent is not comfortable knowing his or her affected status.

There are two approaches to nondisclosing PGT-M: indirect testing and direct testing. Both methods require the use of PGT-A in conjunction with PGT-M. Because chromosome abnormalities are common in embryos, if a patient were to see a report showing that there were embryos not recommended for transfer, they would not be able to know if those embryos were PGT$\mathrm{M}$-abnormal or PGT-A-abnormal. The purpose of this is make it less likely for patients to inadvertently learn their disease status.

Indirect testing excludes embryos that inherited the disease-associated allele from the affected grandparent of the future offspring, without determining whether it was the grandparent's normal or disease-carrying allele. Only embryos that have the allele inherited from the unaffected grandparent would be recommended for transfer. With indirect testing, typically 50\% of embryos would be expected to be rejected as potentially disease-carrying. Patients need to be prepared for the possibility that all tested embryos inherited the allele from the affected grandparent and would not be recommended for use, even though some or all of these embryos may carry the normal allele.

Direct testing is performed by the PGT lab, which tests the patient for the disease under an alias through a clinical laboratory. If the laboratory determines that the patient is affected with the disease, it will proceed with PGT-M and PGT-A to make sure that only disease-free embryos are recommended for use. If the patient is not affected with the disease, only PGT-A will be performed, and embryos will be recommended for transfer based on these results. With this method, someone who is not affected with the disease would not have embryos unnecessarily eliminated from use.

Once the results of nondisclosing PGT-M are complete, the IVF center is informed and the individual or couple undergoes a FET cycle when they are ready to get pregnant. If there are
PGT-normal embryos available, the embryo(s) will be transferred followed by a pregnancy test, which will be given to determine if the FET was successful. If the FET was unsuccessful, and there are additional frozen embryos, they will proceed with FET cycles until they achieve a pregnancy. If there are no additional frozen embryos available, they would need to proceed with a new IVF cycle. In cases in which there are no embryos recommended for use, because of PGT-M or PGT-A results, the patient will have a mock embryo transfer, a FET of no embryos, and a negative pregnancy test. Because a FET can fail to result in a pregnancy even when embryos are transferred, patients should not assume they are affected with the disease in question based on a negative pregnancy test. In this case, the patient will be told that there are no additional frozen embryos and that they will need to proceed with a new IVF cycle.

Although nondisclosing PGT-M is done regularly, it is not without controversy. In giving patients the right not to know their disease status, fertility centers may be performing unnecessary treatments that are costly and carry physical risks. The diseases for which nondisclosing PGT-M is typically performed have significant morbidity with no treatments or cure. Patients at risk for these diseases may choose not to know their status to avoid the stress and other psychological sequelae of knowing they may develop the condition. They may also be concerned about their ability to get health or life insurance should their affected status be known. Regardless of their reasoning, all patients must weigh the risks and benefits to decide the best course of treatment.

\section{PGT for HLA Matching}

PGT for human leukocyte antigen (HLA) typing is used to conceive a child who could donate cord blood or stem cells for transplantation to save an ill sibling (Tur-Kaspa and Jeelani 2015). PGT for this purpose is performed by linkage analysis only. The chance of having an HLA-matched embryo to a full sibling is $25 \%$, assuming that recombination has not occurred. If recombination occurred in an ill child, it would be nearly 
impossible to find a suitable match, as the same recombination event would need to occur again. If the affected child has an autosomal recessive condition, the likelihood of an embryo being PGT-M-unaffected, HLA matched is $18.8 \%$. If the affected child has an $\mathrm{X}$-linked recessive disorder, the likelihood of finding an embryo that is PGT-M-unaffected, HLA-matched is $12.5 \%$.

Those considering PGT for HLA matching need to be counseled regarding the amount of time required for the PGT test to be developed and applied and for an HLA-matched sibling to be born (Tur-Kaspa and Jeelani 2015). They also should be counseled about the possibility of not having any HLA-matched embryos that are PGT-M-normal. Additionally, the transfer of an HLA-matched embryo may not result in pregnancy and live birth. When no HLAmatched embryos are found, patients who want to have more children must decide if they will proceed with additional IVF treatments to try to find a matched embryo, do a FET of an unmatched embryo, or try to conceive naturally.

\section{PGT-M for Mitochondrial Disease}

Mitochondrial diseases can be caused by nuclear DNA mutations or mutations in the mitochondrial DNA (mtDNA) itself. The former can follow any traditional Mendelian inheritance pattern (autosomal recessive, autosomal dominant, or X-linked) and is readily amenable to standard PGT-M. The latter is most commonly maternally inherited, as typically only the oocyte transmits mitochondria and its DNA.

There are multiple biological factors that make mitochondrial inheritance complicated. Heteroplasmy is the presence of both normal and abnormal mtDNA in the same individual. A higher level of abnormal mtDNA increases the chance of an individual being affected with a mitochondrial disorder. However, individuals, including transmitting females, may or may not have clinical symptoms even in the presence of low levels of abnormal mtDNA, depending on tissue distribution. The threshold effect is the percentage of abnormal mtDNA necessary in a given tissue to lead to symptoms of a particular mitochondrial disorder. The bottleneck effect may lead to significant and unpredictable changes in the amount of abnormal mtDNA inherited from one generation to the next.

Options for women at an increased risk of having children with mitochondrial disorders because of mtDNA mutations include prenatal diagnostic testing, PGT-M for mtDNA mutations, and use of an unrelated ovum donor. Another option, although controversial for ethical reasons, and currently not permitted in the United States, is the use of mitochondrial transfer therapy in which a woman has her nuclear DNA transferred into an enucleated oocyte of an unrelated donor, containing the cytoplasm of the ovum donor and thus the donor's mtDNA (Wolf et al. 2015).

PGT-M for mtDNA mutations has significant limitations, as there may be fluctuations in the mtDNA mutation load during embryonic development (Mitalipov et al. 2014; Diot et al. 2016; Craven et al. 2017). Although absence or very low levels of abnormal mtDNA may indicate a reduced likelihood of an embryo being affected with a mitochondrial disorder, the risk cannot be entirely eliminated. Additionally, multiple IVF cycles may be required to find embryos suitable for transfer.

\section{PGT-M for Variants of Uncertain Significance}

With advances in sequencing technology, detection of genetic variants of uncertain significance (VUSs) has increased. When no known pathogenic variant is identified, it is not uncommon for patients to hope that a VUS could explain the disease in their family. PGT-M for a VUS necessitates laboratory approval, as there is liability in testing for a variant that may not be causative of the disease within a given family. Patients need to be cautioned that PGT-M results will only reduce the risk for disease if the VUS is truly causative of the disease in question. If the VUS is not causative, PGT-M will not reduce the risk of that disease in offspring.

\section{GAMETE DONATION}

Gamete donation involves the donation of either oocytes or sperm to assist another individual or 
couple in achieving pregnancy. Oocyte donors are usually between the ages of 21 and 34. Indications for the use of an oocyte donor include women with the following: hypergonadotropic hypogonadism, advanced maternal age, diminished ovarian reserve, poor oocyte/embryo quality, or multiple failed attempts to conceive after fertility treatments. Additional indications include women known to be affected with or carriers of a significant genetic disorder or those who have a family history of a heritable condition for which testing is not available. Oocyte donation is also necessary for male same-sex couples and single men.

Sperm donors are usually under the age of 40. These donors are most frequently obtained from a sperm bank that has a large pool of donors from which recipients may choose. Indications for the use of a sperm donor include the following: azoospermia, severe oligospermia, or other significant sperm or seminal fluid abnormality; ejaculatory dysfunction; or other significant male factor infertility that has not responded to previous attempts at ART. A male may also be affected with or a carrier of a significant genetic disorder or have a family history of a heritable condition for which testing is not available. Additional reasons for use of a sperm donor include an STD that cannot be eradicated or an Rh-positive male with a female partner who is Rh-negative and severely Rh-isoimmunized. Sperm donors are also necessary for women who are trying to conceive without a male partner or female same-sex couples.

\section{Gamete Donor Screening}

The ASRM recommends that donors be in good health with no known genetic abnormalities (Practice Committee of American Society for Reproductive and Practice Committee of Society for Assisted Reproductive 2013). According to guidelines, a complete medical, family, and sexual history should be obtained. Donors need to meet FDA regulations and applicable state tissue banking requirements to reduce the risk of transmitting infectious diseases, and they must also have a complete physical and psychological evaluation.
In addition, the ASRM recommends that a genetic counselor, or other medical professional trained in genetics, perform an assessment of hereditary risk factors for donors (Practice Committee of American Society for Reproductive and Practice Committee of Society for Assisted Reproductive 2013). A complete three-generation pedigree will help determine if there are any known or suspected major Mendelian disorders or conditions with variable expressivity and/or incomplete penetrance, which may put donor-conceived offspring at an increased risk of being affected. If such a condition were identified, the recipient should be informed of the risks so that he/she can make an informed decision regarding use of that donor, or the applicant may be excluded from participating in the donor program. Donors and their first-degree relatives should be free from the following: major malformation with multifactorial or polygenic inheritance, known chromosome abnormalities, and mental retardation of unknown etiology. The ASRM defines major malformation as one that carries serious functional or cosmetic handicap.

According to ASRM guidelines, genetic screening should be performed on all donors, including carrier screening for cystic fibrosis (CF) and other genetic testing as indicated by the donor's ethnic background in accordance with current ASRM recommendations. Chromosome analysis is not required for all donors; it may be performed on all donors as a routine part of a program's screening process to rule out donors with chromosome abnormalities that could affect the success of fertility treatments or may be performed on a donor only if indicated by the donor's medical history.

Because carrier screening will most likely continue to evolve, it should be performed based on professional society recommendations at the time of donation. With the current use of sequencing technology, expanded carrier screening has brought both ease and challenges to the donor selection process.

Prior to the advent of expanded carrier screening, there were a limited number of diseases for which donors could be tested. Given the specific ASRM recommendation for $\mathrm{CF}$ 
screening, it was rare that a donor would not have been screened for the disorder; however, the extent and methodology of screening was variable across donor programs. Some centers would routinely test for spinal muscular atrophy and hemoglobinopathies for all donors, and fragile-X syndrome for oocyte donors. Additional testing may have been based on the donor's ethnicity, such as Tay-Sachs disease testing if the donor were of French Canadian or Cajun ancestry, or as a part of a panel of diseases that were more common in the Ashkenazi Jewish population. Historically, testing was performed by genotyping and, in the case of Tay-Sachs disease, by enzyme analysis. Centers varied in how they managed donors who were identified as carriers for autosomal recessive diseases, with some allowing the use of a donor who was a carrier as long as the recipient partner tested negative for the condition in question. Other centers would disqualify donors who were carriers because they believed that inadequate detection rates of genotyping resulted in unacceptable residual risk in some populations. Gene sequencing and deletion/duplication testing, which would have resulted in lower residual risk, were time-consuming and expensive.

Although not standard, it is now common for many donors to have expanded genetic screening via sequencing methodologies, with or without duplication/deletion analysis. Because it is estimated that an average individual may carry eight to 10 recessive gene mutations (Nussbaum et al. 2007), it is common that people who do expanded testing will learn that they are a carrier of at least one mutation for a disease on a panel. Unless a donor is a carrier of a recessive disease that might have clinical consequences in the heterozygous state (i.e., carriers of ataxia-telangiectasia are at an increased risk of developing cancer) or is an oocyte donor who carries an X-linked recessive disease mutation, most donors are no longer disqualified from donation based solely on carrier status, as gene sequencing now provides a high level of sensitivity to achieve the lowest residual risk for the reproductive partner. If a donor or recipient is a carrier of an autosomal recessive trait, it is important that both parties consider appropriate genetic screen- ing and genetic counseling. Given the significant variability of disease panels within and between laboratories that perform expanded carrier screening, screening results must be carefully reviewed to make sure that appropriate testing has been performed on both the donor and recipient (and/or sperm/egg source).

\section{Types of Gamete Donors}

The majority of gamete donors are anonymous by intent, meaning that the recipient does not know the identity of the donor at the time of donor selection. Although anonymous at the time of donation, donors may agree to varying degrees of contact with donor-conceived individuals once they reach the age of majority. Despite a preference for anonymity by either the donor or the recipient, it is plausible that persons conceived from donor gametes will be able to identify their donors or biologically related individuals and learn that they were conceived via gamete donation. This means that a donor, who believed he or she could remain anonymous, could be identified. Because of the complex nature of gamete donation and advances in genetic medicine, donors and recipients must be aware of the genetic ties that bind them to each other.

Ovum and sperm donors may also be nonanonymous or directed donors. Nonanonymous ovum donors are women found through a donor agency that allows the recipient to know the donor's identity. Directed donors could be the recipient's family member (i.e., sister, brother, aunt, uncle, cousin, niece, or nephew) or friend.

Selecting which type of donor to use is a very personal decision. Use of a relative as a directed donor allows the recipient to have a genetic connection to the child (De Jonge and Barratt 2006). In some cases, an individual selected as a directed donor may be older than the recommended age limit for donation or may harbor a genetic risk that is typically excluded in an anonymous donor program. Recipients must be counseled on the increased risks, such as for aneuploidy in oocytes from women who are of advanced maternal age, and for the risk of new dominant 
mutations, autism, and schizophrenia in offspring from men who are over the age of 40 (Brandt et al. 2019).

\section{Genetic Issues following Gamete Donation}

Changes to the health of donor-conceived offspring, donors themselves, and donor family members can have implications for the health of biologically related individuals. Donors are often asked to notify their gamete donation facilities of changes in their personal or family medical histories. Likewise, when medical issues occur in pregnancies or individuals conceived from gamete donors, it is important that recipient families notify their fertility center and donor facility.

If any new information is believed to have significant implications for the health of related individuals and is clinically relevant or actionable, the gamete donor facility may contact those individuals to whom they believe the information is relevant. This allows the donors and recipients of the donor's gamete to discuss the information with their and their children's personal health-care providers, and to consider pursuing genetic counseling services or other follow-up as applicable to their personal needs and concerns.

\section{SUMMARY AND FUTURE DIRECTIONS}

The landscape of genetics is changing rapidly, particularly in the field of ART. Today, the aim of PGT is to select out embryos that are abnormal. Research is underway using gene editing technologies, such as CRISPR/Cas9, which in the future may allow embryos currently deemed abnormal to be used rather than being discarded or donated to research. Given the multitude of factors necessary for a successful IVF cycle, this will significantly impact the outcome for many patients.

As we identify more candidate genes associated with conditions with complex, multifactorial, and polygenic inheritance, the question of what is appropriate to test for will be a crucial one for IVF centers and patients. Thus, there is a greater need for pre- and posttest counseling in order to fully understand the implications and interpretation of these results. Given the number of genetic issues that may arise in a fertility setting, the expertise of a genetic counselor may prove particularly beneficial for ART clinics.

\section{ACKNOWLEDGMENTS}

The authors thank Justine Witzke, PhD, MPH, CCRP, Alexandra MacWade, MFA, and Pamela Callum, MS, for their editorial comments.

\section{REFERENCES}

Alukal JP, Lamb DJ. 2008. Intracytoplasmic sperm injection (ICSI) - what are the risks? Urol Clin North Am 35: 277288, ix-x. doi:10.1016/j.ucl.2008.01.004

Armstrong S, Akande V. 2013. What is the best treatment option for infertile women aged 40 and over? J Assist Reprod Genet 30: 667-671. doi:10.1007/s10815-0139980-6

Bodurtha J, Strauss JF III. 2012. Genomics and perinatal care. $N$ Engl J Med 366: 64-73. doi:10.1056/ NEJMra1105043

Botting B, Dunnell K. 2000. Trends in fertility and contraception in the last quarter of the 20th century. Popul Trends 32-39.

Brancati F, Mingarelli R, Dallapiccola B. 2003. Recurrent triploidy of maternal origin. Eur J Hum Genet 11: 972 974. doi:10.1038/sj.ejhg.5201076

Brandt JS, Cruz Ithier MA, Rosen T, Ashkinadze E. 2019. Advanced paternal age, infertility, and reproductive risks: a review of the literature. Prenat Diagn 39: 81-87. doi:10 $.1002 / \mathrm{pd} .5402$

Brezina PR, Kutteh WH, Bailey AP, Ke RW. 2016. Preimplantation genetic screening (PGS) is an excellent tool, but not perfect: a guide to counseling patients considering PGS. Fertil Steril 105: 49-50. doi:10.1016/j.fertnstert.2015 .09 .042

Centers for Disease Control and Prevention ASfRM, Society for Assisted Reproductive Technology. 2018. 2016 Assisted reproductive technology fertility clinic success rates report. U.S. Department of Health and Human Services, Atlanta.

Craven L, Tang MX, Gorman GS, De Sutter P, Heindryckx B. 2017. Novel reproductive technologies to prevent mitochondrial disease. Hum Reprod Update 23: 501-519. doi:10.1093/humupd/dmx018

Dahdouh EM, Balayla J, García-Velasco JA. 2015. Comprehensive chromosome screening improves embryo selection: a meta-analysis. Fertil Steril 104: 1503-1512. doi:10 $.1016 /$ j.fertnstert.2015.08.038

De Jonge C, Barratt CL. 2006. Gamete donation: a question of anonymity. Fertil Steril 85: 500-501. doi:10.1016/j .fertnstert.2005.07.1304

Delhanty JD, Griffin DK, Handyside AH, Harper J, Atkinson GH, Pieters MH, Winston RM. 1993. Detection of aneu- 
ploidy and chromosomal mosaicism in human embryos during preimplantation sex determination by fluorescent in situ hybridisation (FISH). Hum Mol Genet 2: 11831185. doi:10.1093/hmg/2.8.1183

Devers PL, Cronister A, Ormond KE, Facio F, Brasington CK, Flodman P. 2013. Noninvasive prenatal testing/noninvasive prenatal diagnosis: the position of the National Society of Genetic Counselors. J Genet Couns 22: 291295. doi:10.1007/s10897-012-9564-0

Diot A, Dombi E, Lodge T, Liao C, Morten K, Carver J, Wells D, Child T, Johnston IG, Williams S, et al. 2016. Modulating mitochondrial quality in disease transmission: towards enabling mitochondrial DNA disease carriers to have healthy children. Biochem Soc Trans 44: 10911100. doi:10.1042/BST20160095

Filges I, Manokhina I, Peñaherrera MS, McFadden DE, Louie K, Nosova E, Friedman JM, Robinson WP. 2015. Recurrent triploidy due to a failure to complete maternal meiosis II: whole-exome sequencing reveals candidate variants. Mol Hum Reprod 21: 339-346. doi:10.1093/ molehr/gaul12

Fishel S. 2018. First in vitro fertilization baby-this is how it happened. Fertil Steril 110: 5-11. doi:10.1016/j.fertnstert .2018 .03 .008

Forabosco A, Percesepe A, Santucci S. 2009. Incidence of non-age-dependent chromosomal abnormalities: a population-based study on 88965 amniocenteses. Eur J Hum Genet 17: 897-903. doi:10.1038/ejhg.2008.265

Fragouli E, Alfarawati S, Daphnis DD, Goodall NN, Mania A, Griffiths T, Gordon A, Wells D. 2011. Cytogenetic analysis of human blastocysts with the use of FISH, CGH and aCGH: scientific data and technical evaluation. Hum Reprod 26: 480-490. doi:10.1093/humrep/deq344

Fragouli E, Alfarawati S, Spath K, Babariya D, Tarozzi N, Borini A, Wells D. 2017. Analysis of implantation and ongoing pregnancy rates following the transfer of mosaic diploid-aneuploid blastocysts. Hum Genet 136: 805-819. doi:10.1007/s00439-017-1797-4

Friedenthal J, Maxwell SM, Munné S, Kramer Y, McCulloh DH, McCaffrey C, Grifo JA. 2018. Next generation sequencing for preimplantation genetic screening improves pregnancy outcomes compared with array comparative genomic hybridization in single thawed euploid embryo transfer cycles. Fertil Steril 109: 627-632. doi:10.1016/j .fertnstert.2017.12.017

Greco E, Minasi MG, Fiorentino F. 2015. Healthy babies after intrauterine transfer of mosaic aneuploid blastocysts. N Engl J Med 373: 2089-2090. doi:10.1056/NEJMc 1500421

Hansen M, Bower C, Milne E, de Klerk N, Kurinczuk JJ. 2005. Assisted reproductive technologies and the risk of birth defects-a systematic review. Hum Reprod 20: 328338. doi:10.1093/humrep/deh593

Harton GL, Cinnioglu C, Fiorentino F. 2017. Current experience concerning mosaic embryos diagnosed during preimplantation genetic screening. Fertil Steril 107: 1113-1119. doi:10.1016/j.fertnstert.2017.03.016

Hassold T, Abruzzo M, Adkins K, Griffin D, Merrill M, Millie E, Saker D, Shen J, Zaragoza M. 1996. Human aneuploidy: incidence, origin, and etiology. Environ $\mathrm{Mol}$ Mutagen 28: 167-175. doi:10.1002/(SICI) 1098-2280 (1996)28:3<167::AID-EM2>3.0.CO;2-B
Heijligers M, van Montfoort A, Meijer-Hoogeveen M, Broekmans F, Bouman K, Homminga I, Dreesen J, Paulussen A, Engelen J, Coonen E, et al. 2018. Perinatal follow-up of children born after preimplantation genetic diagnosis between 1995 and 2014. J Assist Reprod Genet 35: 1995-2002. doi:10.1007/s10815-018-1286-2

Huang B, Prensky L, Thangavelu M, Main D, Wang S. 2004. Three consecutive triploidy pregnancies in a woman: genetic predisposition? Eur J Hum Genet 12: 985-986. doi:10.1038/sj.ejhg.5201274

Kang HJ, Melnick AP, Stewart JD, Xu K, Rosenwaks Z. 2016. Preimplantation genetic screening: who benefits? Fertil Steril 106: 597-602. doi:10.1016/j.fertnstert.2016.04.027

Kimelman D, Confino R, Confino E, Shulman LP, Zhang JX, Pavone ME. 2018. Do patients who achieve pregnancy using IVF-PGS do the recommended genetic diagnostic testing in pregnancy? J Assist Reprod Genet 35: 18811885. doi:10.1007/s10815-018-1289-z

Kurinczuk JJ, Hansen M, Bower C. 2004. The risk of birth defects in children born after assisted reproductive technologies. Curr Opin Obstet Gynecol 16: 201-209. doi:10 .1097/00001703-200406000-00002

Lanzendorf SE, Maloney MK, Veeck LL, Slusser J, Hodgen GD, Rosenwaks Z. 1988. A preclinical evaluation of pronuclear formation by microinjection of human spermatozoa into human oocytes. Fertil Steril 49: 835-842. doi:10.1016/S0015-0282(16)59893-8

Liebaers I, Desmyttere S, Verpoest W, De Rycke M, Staessen C, Sermon K, Devroey P, Haentjens P, Bonduelle M. 2010. Report on a consecutive series of 581 children born after blastomere biopsy for preimplantation genetic diagnosis Hum Reprod 25: 275-282. doi:10.1093/humrep/dep298

Mastenbroek S, Twisk M, van Echten-Arends J, SikkemaRaddatz B, Korevaar JC, Verhoeve HR, Vogel NE, Arts EG, de Vries JW, Bossuyt PM, et al. 2007. In vitro fertilization with preimplantation genetic screening. $N$ Engl $J$ Med 357: 9-17. doi:10.1056/NEJMoa067744

Mastenbroek S, Twisk M, van der Veen F, Repping S. 2011. Preimplantation genetic screening: a systematic review and meta-analysis of RCTs. Hum Reprod Update 17: 454-466. doi:10.1093/humupd/dmr003

Mertzanidou A, Wilton L, Cheng J, Spits C, Vanneste E, Moreau Y, Vermeesch JR, Sermon K. 2013. Microarray analysis reveals abnormal chromosomal complements in over $70 \%$ of 14 normally developing human embryos. Hum Reprod 28: 256-264. doi:10.1093/humrep/des362

Mitalipov S, Amato P, Parry S, Falk MJ. 2014. Limitations of preimplantation genetic diagnosis for mitochondrial DNA diseases. Cell Rep 7: 935-937. doi:10.1016/j.celrep .2014 .05 .004

Munné S, Lee A, Rosenwaks Z, Grifo J, Cohen J. 1993. Fertilization and early embryology: diagnosis of major chromosome aneuploidies in human preimplantation embryos. Hum Reprod 8: 2185-2191. doi:10.1093/oxford journals.humrep.a138001

Munné S, Blazek J, Large M, Martinez-Ortiz PA, Nisson H, Liu E, Tarozzi N, Borini A, Becker A, Zhang J, et al. 2017. Detailed investigation into the cytogenetic constitution and pregnancy outcome of replacing mosaic blastocysts detected with the use of high-resolution next-generation sequencing. Fertil Steril 108: 62-71.e8. doi:10.1016/j .fertnstert.2017.05.002 
D. Lilienthal and M. Cahr

Nussbaum R, McInnes R, Williard H. 2007. Thompson \& Thompson genetics in medicine. Saunders Elsevier, Philadelphia.

Pergament E, Confino E, Zhang JX, Roscetti L, Xien Chen P, Wellman D. 2000. Recurrent triploidy of maternal origin. Prenat Diagn 20: 561-563. doi:10.1002/1097-0223 (200007)20:7<561::AID-PD875>3.0.CO;2-1

Practice Committee of American Society for Reproductive Medicine; Practice Committee of Society for Assisted Reproductive Technology. 2008. Genetic considerations related to intracytoplasmic sperm injection (ICSI). Fertil Steril 90: S182-S184.

Practice Committee of American Society for Reproductive Medicine; Practice Committee of Society for Assisted Reproductive Technology. 2013. Recommendations for gamete and embryo donation: a committee opinion. Fertil Steril 99: 47-62.el. doi:10.1016/j.fertnstert.2012.09.037

Practice Committees of American Society for Reproductive Medicine; Society for Assisted Reproductive Technology. 2013. Mature oocyte cryopreservation: a guideline. Fertil Steril 99: 37-43. doi:10.1016/j.fertnstert.2012.09.028

Practice Committee of the American Society for Reproductive Medicine. 2015a. Diagnostic evaluation of the infertile female: a committee opinion. Fertil Steril 103: e44e50. doi:10.1016/j.fertnstert.2015.03.019

Practice Committee of the American Society for Reproductive Medicine. 2015b. Diagnostic evaluation of the infertile male: a committee opinion. Fertil Steril 103: e18-e25.

Practice Committees of the American Society for Reproductive Medicine and the Society for Assisted Reproductive Technology. 2018. The use of preimplantation genetic testing for aneuploidy (PGT-A): a committee opinion. Fertil Steril 109: 429-436. doi:10.1016/j.fertnstert.2018 .01 .002

Practice Committee of the Society for Assisted Reproductive Technology; Practice Committee of the American Society for Reproductive Medicine. 2008. Preimplantation genetic testing: a Practice Committee opinion. Fertil Steril 90: S136-S143.

Rosenwaks Z, Handyside AH, Fiorentino F, Gleicher N, Paulson RJ, Schattman GL, Scott RT Jr, Summers MC, Treff NR, Xu K. 2018. The pros and cons of preimplantation genetic testing for aneuploidy: clinical and laboratory perspectives. Fertil Steril 110: 353-361. doi:10.1016/j .fertnstert.2018.06.002

Sachdev NM, Maxwell SM, Besser AG, Grifo JA. 2017. Diagnosis and clinical management of embryonic mosaicism. Fertil Steril 107: 6-11. doi:10.1016/j.fertnstert .2016 .10 .006

Scott RT Jr, Upham KM, Forman EJ, Zhao T, Treff NR. 2013. Cleavage-stage biopsy significantly impairs human embryonic implantation potential while blastocyst biopsy does not: a randomized and paired clinical trial. Fertil Steril 100: 624-630. doi:10.1016/j.fertnstert.2013.04.039
Simioni M, Artiguenave F, Meyer V, Sgardioli IC, ViguettiCampos NL, Lopes Monlleó I, Maciel-Guerra AT, Steiner CE, Gil-da-Silva-Lopes VL. 2017. Genomic investigation of balanced chromosomal rearrangements in patients with abnormal phenotypes. Mol Syndromol 8: 187-194. doi:10.1159/000477084

Simon AL, Kiehl M, Fischer E, Proctor JG, Bush MR, Givens C, Rabinowitz M, Demko ZP. 2018. Pregnancy outcomes from more than 1,800 in vitro fertilization cycles with the use of 24-chromosome single-nucleotide polymorphismbased preimplantation genetic testing for aneuploidy. Fertil Steril 110: 113-121. doi:10.1016/j.fertnstert.2018.03 .026

Spinella F, Fiorentino F, Biricik A, Bono S, Ruberti A, Cotroneo E, Baldi M, Cursio E, Minasi MG, Greco E. 2018. Extent of chromosomal mosaicism influences the clinical outcome of in vitro fertilization treatments. Fertil Steril 109: 77-83. doi:10.1016/j.fertnstert.2017.09.025

Steptoe PC, Edwards RG. 1978. Birth after the reimplantation of a human embryo. Lancet 312: 366. doi:10.1016/ S0140-6736(78)92957-4

Stern HJ, Harton GL, Sisson ME, Jones SL, Fallon LA, Thorsell LP, Getlinger ME, Black SH, Schulman JD. 2002. Non-disclosing preimplantation genetic diagnosis for Huntington disease. Prenat Diagn 22: 503-507. doi:10 $.1002 /$ pd.359

Tobler KJ, Brezina PR, Benner AT, Du L, Xu X, Kearns WG. 2014. Two different microarray technologies for preimplantation genetic diagnosis and screening, due to reciprocal translocation imbalances, demonstrate equivalent euploidy and clinical pregnancy rates. J Assist Reprod Genet 31: 843-850. doi:10.1007/s10815-014-0230-3

Treff NR, Levy B, Su J, Northrop LE, Tao X, Scott RT Jr. 2010. SNP microarray-based 24 chromosome aneuploidy screening is significantly more consistent than FISH. Mol Hum Reprod 16: 583-589. doi:10.1093/molehr/ gaq039

Treff NR, Tao X, Schillings WJ, Bergh PA, Scott RT Jr, Levy B. 2011. Use of single nucleotide polymorphism microarrays to distinguish between balanced and normal chromosomes in embryos from a translocation carrier. Fertil Steril 96: e58-e65. doi:10.1016/j.fertnstert.2011.04.038

Tur-Kaspa I, Jeelani R. 2015. Clinical guidelines for IVF with PGD for HLA matching. Reprod Biomed Online 30: 115119. doi:10.1016/j.rbmo.2014.10.007

Verlinsky Y, Cieslak J, Freidine M, Ivakhnenko V, Wolf G, Kovalinskaya L, White M, Lifchez A, Kaplan B, Moise J, et al. 1995. Pregnancies following pre-conception diagnosis of common aneuploidies by fluorescent in-situ hybridization. Hum Reprod 10: 1923-1927. doi:10.1093/oxford journals.humrep.a136207

Wolf DP, Mitalipov N, Mitalipov S. 2015. Mitochondrial replacement therapy in reproductive medicine. Trends Mol Med 21: 68-76. doi:10.1016/j.molmed.2014.12.001 


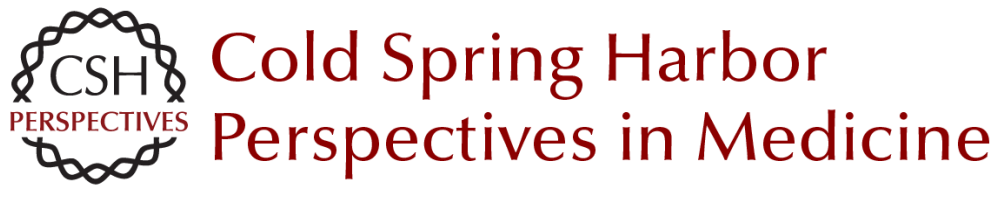

\section{Genetic Counseling and Assisted Reproductive Technologies}

Debra Lilienthal and Michelle Cahr

Cold Spring Harb Perspect Med 2020; doi: 10.1101/cshperspect.a036566 originally published online September 30, 2019

\section{Subject Collection Genetic Counseling: Clinical Practice and Ethical Considerations}

Genetic Risk Assessment in Psychiatry Holly Landrum Peay

Birds of a Feather? Genetic Counseling, Genetic Testing, and Humanism Robert Resta

Bridging the Gap between Scientific Advancement and Real-World Application: Pediatric Genetic Counseling for Common Syndromes and Single-Gene Disorders Julie A. McGlynn and Elinor Langfelder-Schwind

Genetic Counseling, Personalized Medicine, and Precision Health Erica Ramos

Tumor-Based Genetic Testing and Familial Cancer Risk Andrea Forman and Jilliane Sotelo

A Person-Centered Approach to Cardiovascular Genetic Testing Julia Platt

Evidence-Based Genetic Counseling for Psychiatric Disorders: A Road Map Jehannine C. Austin

Supporting Patient Autonomy and Informed Decision-Making in Prenatal Genetic Testing Katie Stoll and Judith Jackson
Impact of Emerging Technologies in Prenatal

Genetic Counseling Blair Stevens

Genetic Counseling and Assisted Reproductive

Technologies Debra Lilienthal and Michelle Cahr

Evolving Roles of Genetic Counselors in the Clinical Laboratory Megan T. Cho and Carrie Guy

Psychological Issues in Managing Families with Inherited Cardiovascular Diseases Jodie Ingles

Informed Consent in the Genomics Era Shannon Rego, Megan E. Grove, Mildred K. Cho, et al.

Predictive Genetic Counseling for

Neurodegenerative Diseases: Past, Present, and Future Jill S. Goldman

Cancer Genetic Counseling--Current Practice and Future Challenges Jaclyn Schienda and Jill Stopfer

Regulating Preimplantation Genetic Testing across the World: A Comparison of International Policy and Ethical Perspectives Margaret E.C. Ginoza and Rosario Isasi

For additional articles in this collection, see http://perspectivesinmedicine.cshlp.org/cgi/collection/ 